\title{
REFERENCES
}

1. M. G. Krein, On certain problems on the maximum and minimum of characteristic values and on the Lyapunov zones of stability, Prikl. Mat. Meh. 15 (1951), 323-348.

2. Z. Nehari, Some eigenvalue estimates, J. Analyse Math. 7 (1959), 79-88.

Carnegie Institute of Technology

\section{WEAK CONVERGENCE OF THE SEQUENCE OF SUCCESSIVE APPROXIMATIONS FOR NONEXPANSIVE MAPPINGS}

BY ZDZISEAW OPIAL

\author{
Communicated by F. Browder, September 26, 1966
}

In a recent paper [4] F. E. Browder and W. V. Petryshyn have shown that if a nonexpansive mapping $T: X \rightarrow X$ of a Hilbert space $X$ into itself is asymptotically regular and has at least one fixed point then, for any $x$ in $X$, a weak limit of a weakly convergent subsequence of the sequence of successive approximations $\left\{T^{n} x\right\}$ is a fixed point of $T$. The main object of the present note is to strengthen considerably this result by showing that under the same assumptions the sequence $\left\{T^{n} x\right\}$ is necessarily weakly convergent.

In $\$ 1$ we recall some basic definitions and prove two simple lemmas. In $\$ 2$ we prove the weak convergence of the sequence $\left\{T^{n} x\right\}$ and in $\$ 3$ we discuss the possibility of the extension of this result to Banach spaces having weakly continuous duality mappings. In $\$ 4$ an application of Theorem 2 stated in $\$ 3$ to a modified sequence of successive approximations is given and, in $\$ 5$, limits of validity of the first key lemma of $\$ 1$ are discussed.

1. Let $C$ be a convex closed set in a Banach space $X$. A mapping $T: C \rightarrow X$ is called nonexpansive if $\|T x-T y\| \leqq\|x-y\|$ for any $x, y$ in $C$. Following [4], a mapping $T: C \rightarrow C$ is said to be asymptotically regular if, for any $x$ in $C$, the sequence $\left\{T^{n+1} x-T^{n} x\right\}=\left\{(I-T)\left(T^{n} x\right)\right\}$ tends to zero as $n \rightarrow \infty$. Finally, a mapping $T: C \rightarrow X$ is called demiclosed if its graph in $C \times X$ is closed in the topology of a Cartesian product induced in $C \times X$ by the weak topology in $C$ and the strong topology in $X$; i.e., if for any sequence $\left\{x_{n}\right\} \subset C$ which converges weakly to an $x_{0}$ in $C$, the strong convergence of the sequence $\left\{T x_{n}\right\}$ to a $y_{0}$ in $X$ implies that $T x_{0}=y_{0}$. 
In a Hilbert space $X$, the following lemma characterizes the weak limit of a weakly convergent sequence:

Lemma 1. If in a Hilbert space $X$ the sequence $\left\{x_{n}\right\}$ is weakly convergent to $x_{0}$, then for any $x \neq x_{0}$,

$$
\liminf _{n \rightarrow \infty}\left\|x_{n}-x\right\|>\liminf _{n \rightarrow \infty}\left\|x_{n}-x_{0}\right\| .
$$

Proof. Since every weakly convergent sequence is necessarily bounded, both limits in (1) are finite. Thus, to prove this inequality, it suffices to observe that in the equality

$$
\begin{aligned}
\left\|x_{n}-x\right\|^{2} & =\left\|x_{n}-x_{0}+x_{0}-x\right\|^{2} \\
& =\left\|x_{n}-x_{0}\right\|^{2}+\left\|x_{0}-x\right\|^{2}+2 \operatorname{Re}\left(x_{n}-x_{0}, x_{0}-x\right)
\end{aligned}
$$

the last term tends to zero as $n$ tends to infinity.

This simple lemma enables us to prove the following useful property of nonexpansive mappings in Hilbert spaces which in [1] has been proved by the means of the theory of monotone mappings.

Lemma 2. In a Hilbert space $X$, for every nonexpansive mapping $T: C \rightarrow X$, the mapping $I-T$ is demiclosed.

Proof. Let $\left\{x_{n}\right\} \subset C$ be a sequence which is weakly convergent to an element $x_{0}$ of $C$ and let the sequence $\left\{x_{n}-T x_{n}\right\}$ converge to an element $y_{0}$ in $X$. Then we have

$$
\liminf _{n \rightarrow \infty}\left\|x_{n}-x_{0}\right\| \geqq \liminf _{n \rightarrow \infty}\left\|T x_{n}-T x_{0}\right\|=\liminf _{n \rightarrow \infty}\left\|x_{n}-y_{0}-T x_{0}\right\|,
$$

so that from Lemma 1 it follows that $x_{0}=y_{0}+T x_{0}$.

2. Using once again Lemma 1 we shall prove the following:

Theorem 1. Let $C$ be a closed convex set in a Hilbert space $X$ and let $T: C \rightarrow C$ be a nonexpansive asymptotically regular mapping for which the set $F$ of fixed points is nonempty. Then, for any $x$ in $C$, the sequence of successive approximations $\left\{T^{n} x\right\}$ is weakly convergent to an element of $F$.

Proof. For every $y$ in $F$, the sequence $\left\{\left\|T^{n} x-y\right\|\right\}$ is nonincreasing, since by the nonexpansivity of $T$ we have

$$
\left\|T^{n+1} x-y\right\|=\left\|T\left(T^{n} x\right)-T y\right\| \leqq\left\|T^{n} x-y\right\| \quad(n=0,1, \cdots) .
$$

Therefore, for any $y$ in $F$, there exists the nonnegative limit

$$
d(y)=\lim _{n \rightarrow \infty}\left\|T^{n} x-y\right\| .
$$


$F$ is a closed convex subset of $C$. For any $d \geqq 0$, the set

$$
F_{d}=\{y \in F: d(y) \leqq d\}
$$

is a convex closed and bounded subset of $F$, nonempty if $d$ is large enough. Since $X$ is a reflexive space, there exists the smallest $\delta$ for which $F_{\delta}$ is nonempty. $F_{\delta}$ consists of exactly one element, say $y_{0}$, since otherwise the midpoint of the segment joining any two distinct elements of $F_{\delta}$ would belong, by the uniform convexity of $X$, to an $F_{d}$ with $d<\delta$.

We shall prove that the sequence $\left\{T^{n} x\right\}$ converges weakly to $y_{0}$. Suppose the contrary. Then, by the reflexivity of $X$ and the boundedness of the sequence $\left\{T^{n} x\right\}$, there exists a weakly convergent subsequence $\left\{T^{n} i x\right\}$ whose limit, say $y$, is different from $y_{0}$. From the asymptotic regularity of $T$, it follows that the sequence $\left\{(I-T)\left(T^{n^{i}} x\right)\right\}$ tends to zero as $n \rightarrow \infty$. Thus, by Lemma 2, we have $(I-T)(y)=0$, i.e. $y$ is a fixed point of $T$. Now, by Lemma 1, we have

$$
\delta=d\left(y_{0}\right)=\lim _{n \rightarrow \infty}\left\|T^{n_{i}} x-y_{0}\right\|>\lim _{n \rightarrow \infty}\left\|T^{n_{i}} x-y\right\|=d(y)
$$

which yields a contradiction with the definition of $\delta$ and completes the proof.

3. From the proof of Theorem 1 it is clear that the assertion of this Theorem may be extended to every Banach space $X$ for which the following four conditions are satisfied: (i) $X$ is reflexive, (ii) the set $F$ of fixed points of a nonexpansive mapping in $X$ is convex, (iii) the set $F_{\delta}$ consists of exactly one element, and (iv) Lemma 1 is valid in $X$.

The first three conditions are satisfied if $X$ is uniformly convex, but, unfortunately, as we shall see in $\$ 5$, Lemma 1 fails to be true for all uniformly convex Banach spaces. However, it remains still valid for a large class of uniformly convex Banach spaces having weakly continuous duality mappings.

Let $X$ be a Banach space, $X^{*}$ its dual space and $(u, x)$ the value of the linear functional $u \in X^{*}$ at the element $x$ of $X$. Let $\mu$ be a continuous strictly increasing real valued function on $R^{+}$with $\mu(0)=0$.

A mapping $J: X \rightarrow X^{*}$ is called (see [3]) a duality mapping of $X$ into $X^{*}$ with the gauge function $\mu$ if both of the following conditions are satisfied:

(a) For every $x$ in $X,(J x, x)=\|J x\| \cdot\|x\|$.

(b) For every $x$ in $X,\|J x\|=\mu(\|x\|)$.

LEMMA 3. If in a Banach space $X$ having a weakly continuous duality mapping $J$ the sequence $\left\{x_{n}\right\}$ is weakly convergent to $x_{0}$, then for any $x$ in $X$ : 


$$
\liminf _{n \rightarrow \infty}\left\|x_{n}-x\right\| \geqq \liminf _{n \rightarrow \infty}\left\|x_{n}-x_{0}\right\|
$$

If, in addition, the space $X$ is uniformly convex, then the equality in (2) occurs if and only if $x=x_{0}$.

Proof. In the relationship

$$
\left(J\left(x_{n}-x_{0}\right), x_{n}-x_{0}\right)=\left(J\left(x_{n}-x_{0}\right), x_{n}-x\right)+\left(J\left(x_{n}-x_{0}\right), x-x_{0}\right)
$$

the last term tends to zero as $n \rightarrow \infty$, since the sequence $\left\{x_{n}-x_{0}\right\}$ converges weakly to zero and therefore, by the weak continuity of $J$, so does the sequence $\left\{J\left(x_{n}-x_{0}\right)\right\}$. Hence, by conditions (a) and (b) of the definition of a duality mapping, we have

$$
\begin{aligned}
\liminf _{n \rightarrow \infty} \mu\left(\left\|x_{n}-x_{0}\right\|\right)\left\|x_{n}-x_{0}\right\| & \leqq \liminf _{n \rightarrow \infty}\left|\left(J\left(x_{n}-x_{0}\right), x_{n}-x\right)\right| \\
& \leqq \liminf _{n \rightarrow \infty}\left\|J\left(x_{n}-x_{0}\right)\right\| \cdot\left\|x_{n}-x\right\| \\
& =\liminf _{n \rightarrow \infty} \mu\left(\left\|x_{n}-x_{0}\right\|\right)\left\|x_{n}-x\right\| .
\end{aligned}
$$

This implies immediately the inequality (2).

To complete the proof, it suffices to observe that if, in a uniformly convex Banach space $X$, both limits in (2) were equal for an $x$ different from $x_{0}$, then for the midpoint $y$ of the segment joining $x$ to $x_{0}$ we would have the inequality

$$
\liminf _{n \rightarrow \infty}\left\|x_{n}-y\right\|<\liminf _{n \rightarrow \infty}\left\|x_{n}-x_{0}\right\|
$$

which is impossible.

Now it is clear that using Lemma 3 in the place of Lemma 1 we are able to prove an extended version of Lemma 2 obtained by replaing the assumption that $X$ is a Hilbert space by the assumption that $X$ is a uniformly convex Banach space having a weakly continuous duality mapping. In the case $C=X$ this statement has been proved by F. E. Browder in [3] even in a more general setting - for all real (not necessarily uniformly convex) Banach spaces with a weakly continuous duality mapping. His proof, however, rests heavily upon a connection between nonexpansive mappings and the so-called $J$ monotone mappings, a connection which works well only for nonexpansive mappings defined on the whole space $X$. In Hilbert spaces this restriction may be easily lifted by an application of the Kirszbraun-Valentine theorem (see [1]) on the existence of extensions of nonexpansive mappings, but in arbitrary Banach spaces such an 
extension theorem is not available, and the question of how to eliminate this restriction remains open.

At any rate, from Lemma 3 and the corresponding generalized version of Lemma 2, we obtain the following theorem which may be proved along the same line as Theorem 1:

THEOREM 2. Let $C$ be a closed convex set in a uniformly convex Banach space $X$ having a weakly continuous duality mapping. Assume that the nonexpansive asymptotically regular mapping $T: C \rightarrow C$ has in $C$ at least one fixed point. Then for any $x$ in $C$, the sequence $\left\{T^{n} x\right\}$ is weakly convergent to a fixed point of $T$.

Let us observe that Theorem 2 applies in particular to the spaces $1^{p}$, for $1<p<+\infty$, since all these spaces are uniformly convex and have (see [3]) weakly continuous duality mappings.

4. For a given nonexpansive mapping $T$ of a closed convex set $C$ of a Banach space $X$ into itself and any $\lambda \in(0,1)$, the mapping $T_{\lambda}=\lambda I+(1-\lambda) T$ is nonexpansive, maps $C$ into itself and has the same set of fixed points as T. Moreover, as it has been pointed out by M. A. Krasnosel'skiǐ [6] for $\lambda=\frac{1}{2}$ and by H. Schaefer [7] for an arbitrary $\lambda$, if $X$ is uniformly convex and $T$ has at least one fixed point in $C$, then the mapping $T_{\lambda}$ is asymptotically regular (see also [4]). Combining these results along with Theorem 2 we obtain the following:

Theorem 3. Let $C$ be a closed convex set in a uniformly convex Banach space $X$ having a weakly continuous duality mapping and let $T: C \rightarrow C$ be a nonexpansive mapping with at least one fixed point. Then for any $x$ in $C$ and any $\lambda \in(0,1)$ the sequence of successive approximations $\left\{T_{\lambda}^{n} x\right\}$ is weakly convergent to a fixed point of $T$.

For weakly continuous nonexpansive mappings in real Hilbert spaces, Theorem 3 has been proved by H. Schaefer in [7] by a geometrical argument which contains in a somewhat crude form all essential ideas of our proof of Theorem 1.

It should be noticed that Theorem 3 gives a partial affirmation of a conjecture of $H$. Schaefer, that for weakly continuous nonexpansive mappings $T$ in uniformly convex Banach spaces the sequence of successive approximations constructed by the aid of the auxiliary mapping $T_{\lambda}(0<\lambda<1)$ is always weakly convergent. Namely, from this theorem it follows that it is really so for Banach spaces with weakly continuous duality mappings. But, as there exist (see $\$ 5$ below) uniformly convex spaces that do not have weakly continuous duality mappings, the question as to whether Schaefer's conjecture in its original form is true or not still remains open. 
Let us recall that if the set $C$ is bounded, then the existence of at least one fixed point of a nonexpansive mapping follows from a theorem proved independently by F. E. Browder [2] and W. A. Kirk [5].

5. The key role played by Lemma 1 in the proofs of Lemma 2 and Theorem 1 raises a natural question as to what extent this characterization of the weak limit by the norm is valid in general Banach spaces. A partial positive answer to this question is given by Lemma 3. However, we shall show that Lemma 1 cannot be extended to the broader class of all uniformly convex Banach spaces. We shall prove that, for $1<p<2$ and $2<p<+\infty$, none of the spaces $L^{p}[0,2 \pi]$ have the property stated in that lemma. By Lemma 3 , this will give an indirect proof that none of these spaces have a weakly continuous duality mapping (for $p=4$ this has been explicitly proved in [3]).

Let $\phi$ be a periodic real valued function of period $2 \pi$ such that

$$
\begin{aligned}
\phi(t) & =1 & & \text { for } 0 \leqq t \leqq(3 / 4) \pi \\
& =-2 & & \text { for }(3 / 4) \pi<t<2 \pi .
\end{aligned}
$$

Since for every step function $\psi$ we have

$$
\lim _{n \rightarrow \infty} \int_{0}^{2 \pi} \phi(n t) \psi(t) d t=0
$$

the sequence $\{\phi(n t)\}$ is weakly convergent to zero in each of the spaces $L^{p}[0,2 \pi](1<p<+\infty)$. For any constant function equal to $c$, we have

$$
\begin{aligned}
\Phi_{p}(c) & =\lim _{n \rightarrow \infty}\|\phi(n t)-c\|^{p}=\lim _{n \rightarrow \infty} \int_{0}^{2 \pi}|\phi(n t)-c|^{p} d t \\
& =\int_{0}^{2 \pi}|\phi(t)-c|^{p} d t .
\end{aligned}
$$

Hence

$$
\Phi_{p}^{\prime}(0)=-p \int_{0}^{2 \pi}|\phi(t)|^{p-1} \operatorname{sgn} \phi(t) d t .
$$

By the definition of $\phi, \Phi_{p}^{\prime}(0) \neq 0$ whenever $p \neq 2$. This implies that $\Phi_{p}(0)$ is not an extremal value of the function $\Phi_{p}(c)$, except for the case $p=2$. 


\section{REFERENCES}

1. F. E. Browder, Fixed-point theorems for noncompact mappings in Hilbert space, Proc. Nat. Acad. Sci. U.S.A. 53 (1965), 1272-1276.

2. - Nonexpansive nonlinear operators in a Banach space, Proc. Nat. Acad. Sci. U.S.A. 54 (1965), 1041-1044.

3. - Fixed point theorems for nonlinear semicontractive mappings in Banach spaces, Arch. Rational Mech. Anal. 21 (1966), 259-269.

4. - W. V. Petryshyn, The solution by iteration of nonlinear functional equations in Banach spaces, Bull. Amer. Math. Soc. 72 (1966), 571-575.

5. W. A. Kirk, A fixed point theorem for mappings which do not increase distance, Amer. Math. Monthly 72 (1965), 1004-1006.

6. M. A. Krasnosel'skiY, Two observations about the method of successive approximations, Uspehi Mat. Nauk 10 (1955), no. 1 (63), 123-127 (Russian).

7. H. Schaefer, Über die Methode sukzessiver Approximationen, Jber. Deutsch. Math.-Verein. 59 (1957), 131-140.

BROWN UNIVERSITY AND

Jagellonian University, Krak6w, Poland 